\title{
Eurasian Journal of Physics and Functional Materials
}

$2021,5(4), 218-228$

\section{The features of deformation-stimulated $\mathrm{RbI}$ luminescence}

\author{
L.N. Myasnikova*, A.G. Maratova, K.Sh. Shunkeyev \\ K. Zhubanov Aktobe Regional University, Aktobe, Kazakhstan \\ E-mail: myasnikova_ln@mail.ru
}

DOI: $10.32523 /$ ejpfm.2021050406

Received: 07.11.2021 - after revision

\begin{abstract}
This paper studies deformation-stimulated features of radiative relaxation of self-trapped excitons and recombination assembly of exciton-like luminescence in RbI crystal. Methods of research were luminescence and thermal activation spectroscopy. The identity of the mechanism of manifestation of the $\mathrm{X}$-ray luminescence, tunnel luminescence and thermally stimulated luminescence spectra were found in the elastically deformed $\mathrm{RbI}$ crystal, interpreted by the luminescence of self-trapped exciton, tunnel recharge of $F^{\prime}, V_{K}$-pairs and thermally stimulated recombination of $e^{-}, V_{K}$-centers, respectively. The temperatures of the maximum destruction peaks of thermally stimulated luminescence, their spectral composition and activation energies were determined experimentally, on the basis of which the mechanisms of recombination assembly of exciton-like luminescences in a RbI crystal were interpreted. Uniaxial elastic deformation leads to the effective formation of point radiation defects $\left(F^{\prime}, H_{A}, V_{K}\right.$ centers) in comparison with an unbroken lattice, where the predominant mechanism is the association of interstitial atoms ( $H$-centers) with the formation of $I_{3}^{-}$-centers.
\end{abstract}

Keywords: RbI crystal, X-ray luminescence, tunnel luminescence, thermostimulated luminescence, uniaxial elastic deformation.

\section{Introduction}

The reliably established mechanisms of the manifestation of the intrinsic luminescence of alkali halide crystals (AHCs), which is carried out through a self-trapped exciton state [1-3], formed due to the deformation of the crystal lattice, provides the widest opportunities for further investigation of the radiative relaxation of exciton-like structures with a decrease in the lattice symmetry [4-7]. 
In AHCs, the molecular two-center structure of self-trapped excitons (STEs) is very dependent (sensitive) on the symmetrical arrangement of crystal-forming particles, and the slightest decrease in the lattice symmetry increases the probability of an unstable state of STEs ending in their decay, mainly through two channels: primary radiation defects and luminescence [8-11].

Local deformations due to the difference in the sizes of both anionic and cationic homologues $[2,5]$, point vacancy and radiation defects [12-13], as well as uniaxial elastic deformation $[6,11,14]$ have traditionally been used as reducing the symmetry of the lattice of AHCs.

In the experimental plan, elastic deformation is successfully used not only as a method of influencing the relaxation processes of STEs, but also for recognizing the nature of luminescence in AHCs [15]. For the first time, low-temperature uniaxial elastic deformation was used as an external perturbing factor that reduces the lattice symmetry to solve the problem of the intrinsic or impurity nature of $E_{x}$-luminescence in $\mathrm{KI}$ and $\mathrm{RbI}$ crystals [16]. The intensity of intrinsic luminescence of exciton nature correlates with an increase in the degree of elastic deformation, and the intensity of luminescence of impurity nature, on the contrary, will decrease until it disappears, since the transfer of exciton energy to impurities will worsen due to the inclusion of the mechanism of their self-trapping in regular lattice sites [15].

Thus, the directed effect on the predisposing state of anionic excitons opens up the possibility of studying the radiative relaxation of electronic excitations in order to develop materials with specified optical characteristics, for example, as high-speed scintillation detectors based on AHCs [17-18].

\section{Experimental technique and research objects}

The multifunctional spectral complex performs scanning of $X$-ray luminescence, tunnel luminescence (TL) and thermostimulated luminescence spectra (TSL), as well as integrated scanning of TL and TSL crystals using a high-power monochromator MSD-2 and a photoelectronic multiplier of H 8259 type of the company "Hamamatsu", operating in the photon counting mode, controlled by special programs SpectraScan and ThermoScan in a wide spectrum range of $200 \div 850 \mathrm{~nm}$ and temperatures of $85 \div 400 \mathrm{~K}$ under the influence of uniaxial deformation ( $\varepsilon=0.1-1.2 \%$ ) under high technical vacuum conditions [19].

During scanning of each TSL spectrum in the specified spectral range, the sample temperature changed by $1.5 \div 2.0 \mathrm{~K}$, i.e. it practically remains as stationary radiation during scanning at a maximum speed $(50 \mathrm{~nm} / \mathrm{s})$. TSL of the irradiated crystals was recorded when heated at a constant rate $\beta=0.15 \mathrm{deg} / \mathrm{s}$. The crystals were irradiated with ionizing radiation using the RUP-120 X-ray machine. The operating mode $(\mathrm{W}, 3 \mathrm{~mA}, 100 \mathrm{kV})$ of the installation practically does not lead to the creation of radiation defects that distort the $X$-ray luminescence spectra of crystals. The optical transparency of the spectral range $(2.0 \div 6.0 \mathrm{eV})$ is also ensured by the fact that the maximum of the absorption band of the $F$-center $(1.87 \mathrm{eV})$ in $\mathrm{RbI}$ crystal is outside the studied region of the radiation spectrum. 
$\mathrm{RbI}$ single crystals were grown at the Institute of Physics of the University of Tartu using the Kiropoulos method in an atmosphere of inert helium from specially purified raw materials [20].

\section{Experimental results and discussion}

It is known that three STEs bands were registered in the X-ray and photoluminescence spectra at a temperature of $4.2 \mathrm{~K}$ for $\mathrm{RbI}$ crystal, the maxima of which are located at $3.9 \mathrm{eV}(\sigma)$ and $2.3 \mathrm{eV}(\pi)$, as well as $3.1 \mathrm{eV}\left(E_{x}\right)[1-2,8-10]$. The symbols $\sigma$ and $\pi$ indicate the polarization of the STEs radiation.

To date, the nature of $E_{x}$-luminescence in $\mathrm{RbI}$ and $\mathrm{KI}$ crystals has been actively discussed, or at least there are two points of view. Most researchers believe that $E_{x}$-luminescence is an intrinsic luminescence of STEs with a weak-off configuration $[8-9,16,21]$, and there is also an opinion that $E_{x}$-luminescence refers to the emission of electron excitation involving a light sodium cation in $\mathrm{RbI}$ lattice [11, 22-23].

The effect of uniaxial elastic deformation on the X-ray luminescence spectra at $85 \mathrm{~K}$ is shown in Figure 1.

The boundary of elastic and plastic deformation is determined experimentally by the linear section of the dependence of the intensity of $\sigma$ - and $E_{x}$ luminescence on the degree of deformation at which Hooke's law is valid [16]. In this regard, to create only the elastic part of the deformation, the crystals were deformed at low temperature $(85 \mathrm{~K})$ within the range of $\varepsilon=0.8-1.0 \%$ in a special cryostat under high technical vacuum conditions [19].

It follows from Figure 1 that in the absence of deformation in $\mathrm{RbI} X$-ray luminescence spectra at $85 \mathrm{~K}$, one band of radiation with a maximum at $3.9 \mathrm{eV}$, related to $\sigma$-luminescence, was extinguished compared to the intensity at $4.2 \mathrm{~K}$ (curve 1). At the same time, the intensities of the luminescence band with maxima at $3.1 \mathrm{eV}\left(E_{x}\right)$ and $2.3 \mathrm{eV}(\pi)$, which were recorded at $4.2 \mathrm{~K}$, are at the limit of the sensitivity of the equipment due to temperature quenching. The intensity ratios of the luminescence bands with maxima at $3.9 \mathrm{eV}(\sigma), 3.1 \mathrm{eV}\left(E_{x}\right)$ and $2.3 \mathrm{eV}(\pi)$ in the operating temperature mode of the equipment $(85 \mathrm{~K})$ are approximately $1: 0.04: 0.007$, respectively.

With an increase in the temperature of $\mathrm{RbI}$ sample from $4.2 \rightarrow 60 \mathrm{~K}$, the intensity of $\pi$-luminescence $(2.3 \mathrm{eV})$ increases, and the intensity of $E_{x}$-luminescence $(3.1 \mathrm{eV})$ decreases, and their temperature dependences resemble a $\chi$-shaped course. After $60 \mathrm{~K}$, the luminescence intensity of both bands drops sharply and an insignificant part remains, and at $80 \mathrm{~K}$, a single $\sigma$-luminescence band $(3.9 \mathrm{eV})$ remains dominant.

It is known that in all AHCs, with an increase in temperature $(4.2 \rightarrow 80 \mathrm{~K})$, the luminescence of STEs undergoes temperature quenching, as a result of which the quantum yield of luminescence sharply decreases, and, accordingly, the radiation intensity decreases by more than an order of magnitude, when compared with the intensity at $4.2 \mathrm{~K}$ [24].

It follows from Figure 1 that the intensity of $\sigma$-luminescence $(3.9 \mathrm{eV})$ increases 


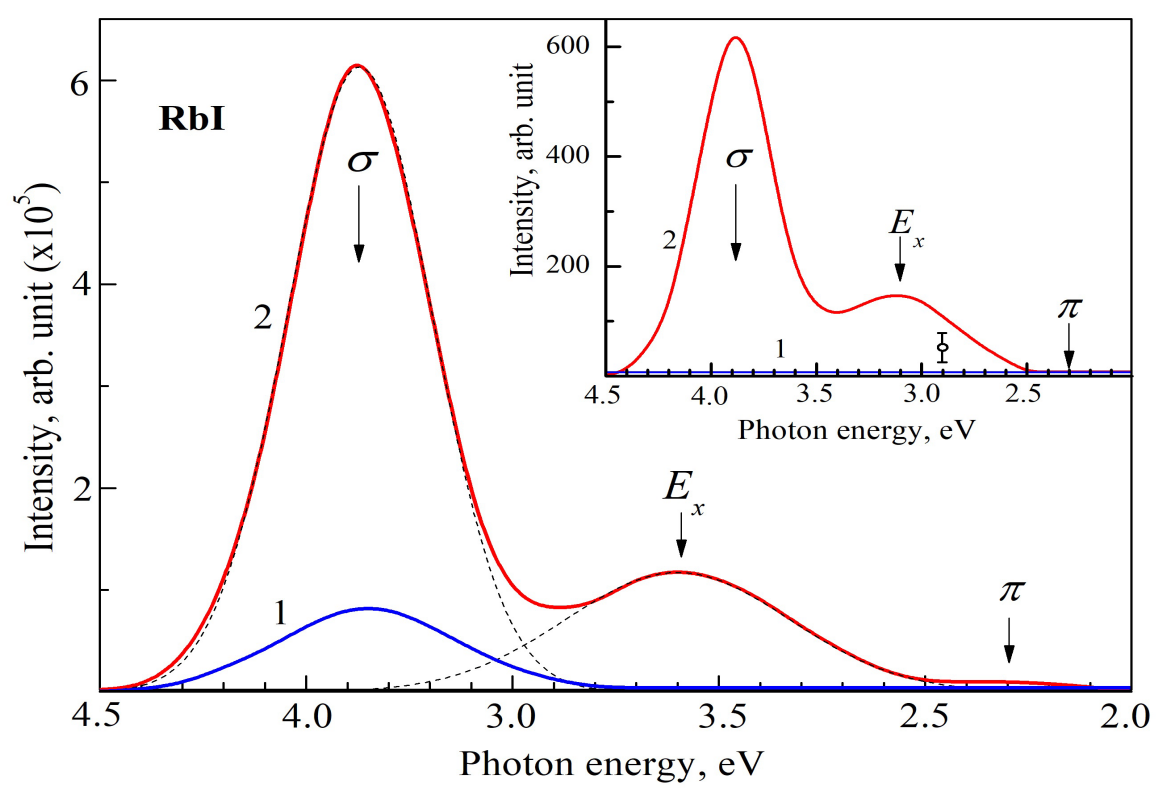

Figure 1. X-ray spectra of RbI crystal measured at $85 \mathrm{~K}$ in the absence of deformation (curve 1) and at uniaxial elastic deformation $\varepsilon=0.8-1.0 \%$ (curve 2). The dashed lines show the components of the spectrum decomposition into elementary components. On the insert - TL in the absence of deformation (curve 1) and with uniaxial elastic deformation $\varepsilon=0.8 \div 1.0 \%$ (curve 2 ) of $\mathrm{RbI}$ crystal, after irradiation with X-rays for 30 minutes at $85 \mathrm{~K}$.

by more than 8 times in an elastic-deformed $\mathrm{RbI}$ crystal at $85 \mathrm{~K}$, in addition, an increase in the intensity of $E_{x}(3.1 \mathrm{eV})$ and $\pi(2.3 \mathrm{eV})$ - luminescence is recorded.

Based on the correlated increase in the intensity of $E_{x}$ - and $\sigma$-luminescence with a decrease in the symmetry of the lattice of RbI crystal, it is concluded that the nature of $E_{x}$-luminescence is not associated with the participation of sodium impurity, as many researchers assumed [8-9, 21]. The experimental results obtained in [16] allow us to assume that their $E_{x}$-luminescence corresponds to the radiative decay of STEs, which has its own nature in RbI crystal.

The mechanism of the appearance of intense luminescence of STEs in an elastically deformed $\mathrm{RbI}$ crystal is possible during successive relaxation stages of high-energy electron-hole pairs created by $X$-ray radiation during recombination of which free excitons are formed. It is experimentally shown that the subsequent relaxation of free excitons with high efficiency occurs from the self-trapping state in regular lattice nodes with characteristic intrinsic luminescence, according to the following scheme:

$$
e^{-}+e^{+} \rightarrow e_{F}^{0} \rightarrow e_{s}^{0} \rightarrow h \nu\left(\sigma, E_{x}, \pi\right) .
$$

According to the proposed scheme, the increase in the intensity of STEs luminescence in the AHCs occurs due to a reduction in the free path of unrelaxed excitons before self-trapping, which will lead to an increase in the probability of self-trapping in regular lattice nodes [15].

In this regard, uniaxial elastic deformation, carried out at $85 \mathrm{~K}$, is a very effective experimental method for separating the luminescence of AHCs that have their own or impurity nature.

In this regard, we have registered the spectrum of tunnel luminescence of an elastically deformed RbI crystal at $85 \mathrm{~K}$ (in the box curve 2 of Figure 1) in spectral composition, which coincides with the spectrum of X-ray luminescence (Figure 1). 
The maxima of $\sigma(3.9 \mathrm{eV})$ and $E_{x}(3.1 \mathrm{eV})$ luminescence, which are absent in the undeformed $\mathrm{RbI}$ crystal, are clearly distinguished. As a rule, tunnel luminescence is studied at low temperatures $(4.2 \mathrm{~K})$, when nearby charged radiation defects are created that are capable of tunnel recharging. To do this, it is necessary to create a sufficient concentration of tunneling pairs of radiation defects by irradiation with ionizing radiation. In our case, $\mathrm{RbI}$ crystals, both not deformed and elastically deformed, were irradiated with $\mathrm{X}$-ray radiation in the isodose mode at $85 \mathrm{~K}$ for 30 minutes.

The coincidence of the spectral composition of the RL and TL of the elastically deformed RbI crystal shows that the final stage of radiative relaxation is the selftrapping state of the anionic exciton, similar to scheme (1), with tunnel recharge of $F^{\prime}, V_{K}$-pairs:

$$
F^{\prime}, V_{K} \rightarrow v_{a}^{-} e^{-} e^{-}+e_{s}^{+} \rightarrow F \ldots \ldots\left(e^{-}+e_{s}^{+}\right) \rightarrow e_{s}^{0} \rightarrow h v\left(\sigma, E_{x}, \pi\right) .
$$

Since the $F^{\prime}\left(v_{a}^{-} e^{-} e^{-}\right)$-center has a weakly bound electron, since 2 electrons are localized in one anion node of the lattice, it is quite possible for an electron to tunnel from the $F^{\prime}$-center to the $V_{K}$-center, which represents a self-trapped hole, at elevated concentrations of $F^{\prime}, V_{K}$-pairs. As a result, STEs is formed by recombination with radiative relaxation of $\sigma(3.9 \mathrm{eV})$ and $E_{x}(3.1 \mathrm{eV})$-luminescence.

Apparently, an increased concentration of tunneling pairs of radiation defects is created during elastic deformation, since in the absence of deformation, due to the weak intensity, it is not possible to register structural luminescence bands.

In this regard, studies of integral thermally stimulated luminescence, especially the TSL spectra of elastically deformed RbI crystals, are very informative.

Figure 2 shows the curves of thermally stimulated luminescence of RbI crystal in the absence of deformation (curve 1) and at uniaxial elastic deformation $(\varepsilon=0.8-1.0 \%$ ) carried out at $85 \mathrm{~K}$ (curve 2 ). To ensure a correct comparison of the experimental results, the crystals were irradiated with X-rays under the same conditions in the isodose mode with an exposure of 30 minutes at $85 \mathrm{~K}$. It follows from Figure 2 that in the absence of deformation, TSL peaks with maxima were recorded at temperatures of $113 \mathrm{~K}\left(F^{\prime}\right), 128 \mathrm{~K}\left(H_{A}\right), 148 \mathrm{~K}\left(V_{K}\right)$, $178 \mathrm{~K}\left(V_{K A}\right)$ and $360 \mathrm{~K}\left(I_{3}^{-}\right)$. The symbols of the corresponding radiation defects that are thermally destructive in the specified temperature values are shown in parentheses. In the absence of deformation, except for low-temperature radiation defects ( $F^{\prime}, H_{A}$ and $V_{K}$-families), $I_{3}^{-}$-centers are effectively created, as is known, with the association of mobile interstitial halogen atoms ( $H$-centers). Low-temperature elastic deformation radically changes the ratio of the dominant peaks of TSL: first, the high-temperature peak of TSL at $360 \mathrm{~K}$, corresponding to the thermal destruction of the $I_{3}^{-}$-centers, completely disappears, and secondly, more intense low-temperature peaks of the above-mentioned radiation defects appear (compare curves 1 and 2 of Figure 2).

Thus, a redistribution of the intensity of high-temperature TSL peaks (curve 1 of Figure 2) in favor of low-temperature peaks (curve 2 of Figure 2), accompanied by the effective formation of single hole ( $H_{A}$ and $V_{K}$-families) and electronic $\left(F^{\prime}\right)$-color centers, instead of the high-temperature $I_{3}^{-}$-center, was found. This effect is apparently associated with a decrease in the probability of long - range 
interactions of mobile halogen atoms with the formation of three-halide radiation defects $-I_{3}^{-}$-centers in elastically deformed RbI crystals.

Thus, in the experimental plan, a unique situation is created for registering the spectra of integrated thermally stimulated luminescence of elastically deformed crystals, since they have become intense.

A comparative analysis of the TSL curves (curves 1 and 2 of Figure 2) shows that at a low-temperature $(85 \mathrm{~K})$ uniaxial elastic deformation, the intensity of the peaks of the $F^{\prime}$-centers increases by more than 8 times, $V_{K}$ and $V_{K A}$-centers by more than 4 times compared to the unbroken lattice of RbI crystal. The increase in the intensity of the $V_{K}$-centers is relatively low compared to the $F^{\prime}$-centers, due to the fact that during the thermal destruction of the $F^{\prime}$-centers $(113 \mathrm{~K})$, the electrons released from it recombine with self-trapped holes ( $V_{K}$-centers), forming an exciton-like formation. As a result, even before the destruction temperature is reached, the concentration of stationary $V_{K}$-centers decreases at $113 \mathrm{~K}$ due to recombination with electrons released from the $F^{\prime}$-centers.

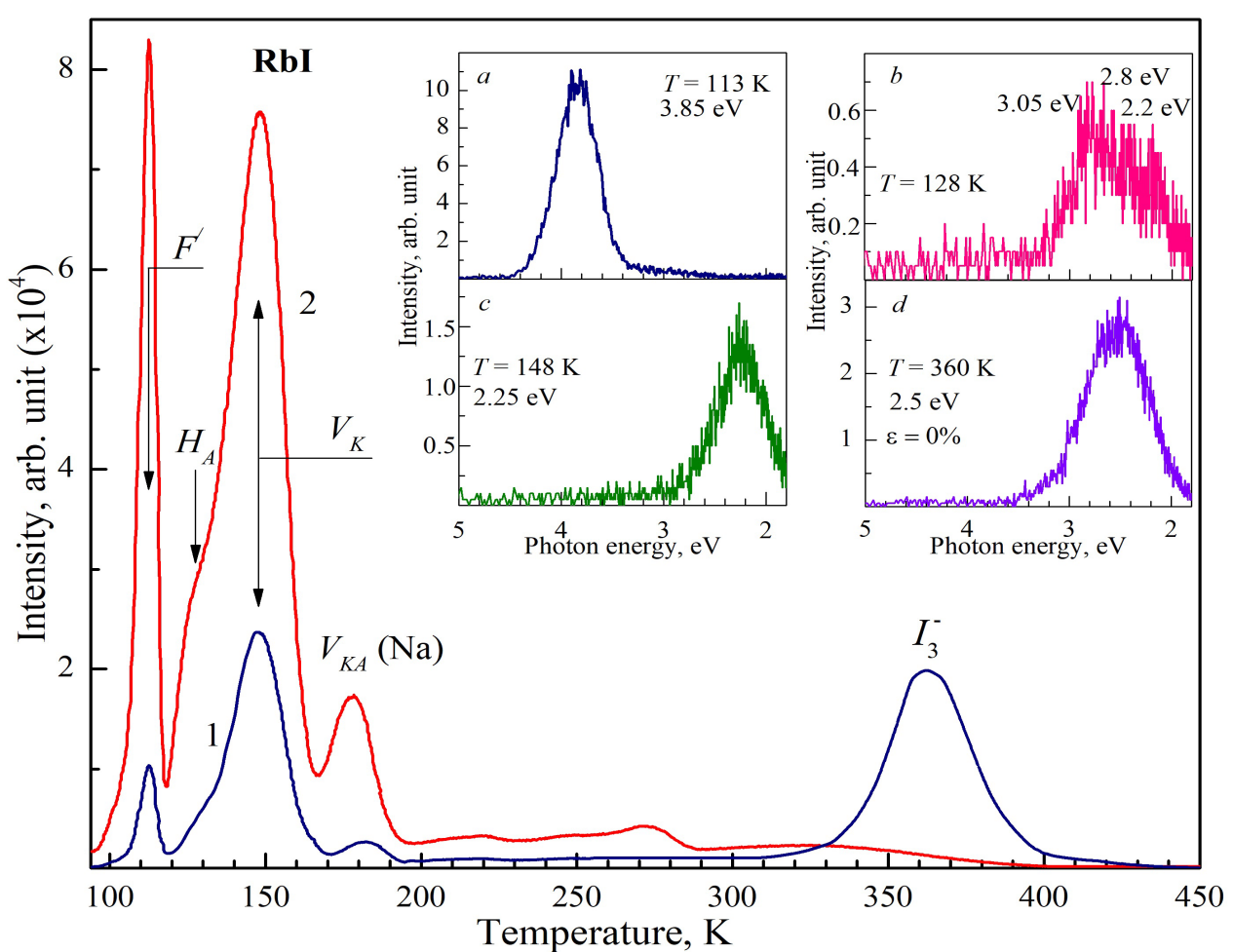

Figure 2. Integral thermostimulated luminescence before (curve 1) and at (curve 2) low-temperature deformation and TSL spectra $(a, b, c, d)$ of RbI crystal irradiated in the isodose mode (30 minutes) at $85 \mathrm{~K} . a, b, c, d$ are the TSL spectra at $113 \mathrm{~K}, 128 \mathrm{~K}, 148 \mathrm{~K}$ and $360 \mathrm{~K}$, respectively.

So, at $113 \mathrm{~K}$, the maximum dissociation of $F^{\prime}$-centers occurs with the formation of free electrons $\left(e^{-}\right)$and $F$-centers according to the following scheme:

$$
F^{\prime}\left(v_{a}^{-} e^{-} e^{-}\right) \rightarrow F\left(v_{a}^{-} e^{-}\right) \ldots . e^{-} .
$$

At the same time, the concentration of $F$-centers, thermally stable up to $400 \mathrm{~K}$, and free electrons in the conduction band increases in the crystal, which are more likely to recombine with fixed self-trapped holes ( $V_{K}$-centers) according to the following scheme:

$$
e^{-} \ldots e_{s}^{+}\left(V_{K}-\text { center }\right) \rightarrow e_{s}^{0} \rightarrow h v \rightarrow(\sigma, \pi) .
$$


Since the recombination of free electrons with $V_{K}$-centers occurs from the conduction band, it is reasonable to expect the appearance of mainly $\sigma$-luminescence with maxima at $3.85 \mathrm{eV}$. Indeed, the spectral composition of the TSL peak at $113 \mathrm{~K}$, when the $F^{\prime}$-centers are destroyed as much as possible, consists of one radiation band with a maximum at $3.85 \mathrm{eV}$, corresponding to $\sigma$-luminescence (box a, Figure 2). The absence of $\pi$-luminescence with a maximum at $2.3 \mathrm{eV}$ is apparently due to temperature quenching at $113 \mathrm{~K}$, since it is quenched already at $75 \mathrm{~K}$.

In the temperature range of $120-130 \mathrm{~K}$, the TSL peak was registered, the spectral composition of which consists of three non-elementary radiation bands with maxima at $3.05 \mathrm{eV}, 2.8 \mathrm{eV}$ and $2.2 \mathrm{eV}$. It is reasonable to associate the last band of radiation $(2.2 \mathrm{eV})$ with the $\alpha$-luminescence band $(2.22 \mathrm{eV})$ of STE in $\mathrm{RbI}$ crystal, since the $\pi$-luminescence $(2.3 \mathrm{eV})$ is extinguished in the specified temperature range.

Analysis of the X-ray and TL spectra of RbI crystal shows that during elastic deformation, the intrinsic $\sigma$ - and $E_{x}$-luminescence increase with maxima at $3.9 \mathrm{eV}$ and $3.1 \mathrm{eV}$, respectively (Figure 1). If we take into account that similar TSL spectra were not recorded in the absence of deformation, it can be assumed that the radiation with a maximum at $3.05 \mathrm{eV}$ is the result of deformation-stimulated recombination luminescence in $\mathrm{RbI}$ crystals. As for the radiation with a maximum at $2.8 \mathrm{eV}$ in the TSL spectra, its nature has not yet been established.

The maximum TSL in the temperature range of $145-148 \mathrm{~K}$ corresponds to the thermal delocalization of $V_{K}$-centers. In this case, we should expect recombination of mobile holes with $F$-centers with the formation of exciton-like luminescence in the anionic vacancy field, according to the proposed scheme:

$$
e^{+}+v_{a}^{+} e^{-} \rightarrow e_{s}^{0}\left(v_{a}^{+}\right) \rightarrow \alpha-\text { luminescence. }
$$

Indeed, in the TSL spectra in the specified temperature range, radiation with a maximum at $2.25 \mathrm{eV}$, the so-called $\alpha$-luminescence, is registered. It is known that $\alpha$ and $\pi$ luminescences have very close luminescence bands with maxima at $2.22 \mathrm{eV}$ and $2.26 \mathrm{eV}$, respectively. It follows from this that during recombination of mobile holes with $F$-centers, a non-elementary radiation band may be registered in the TSL spectra, depending on how the anionic vacancy field affects $\left(v_{a}^{+}\right)$.

If there is no influence of $v_{a}^{+}$on the process of recombination of $V_{K}$ - and $F$-centers, then we should register $\pi$-luminescence:

$$
e^{+}+v_{a}^{+} e^{-} \rightarrow v_{a}^{+} \ldots \ldots e_{s}^{0} \rightarrow \pi-\text { luminescence. }
$$

If we take into account the influence of $v_{a}^{+}$on the process of recombination of $V_{K}$ - and $F$-centers, then we register $\alpha$-luminescence:

$$
e^{+}+v_{a}^{+} e^{-} \rightarrow e_{s}^{0}\left(v_{a}^{+}\right) \rightarrow \alpha-\text { luminescence. }
$$

Apparently, radiation with a maximum at $2.25 \mathrm{eV}$ is recorded in the TSL spectra during thermal destruction of the $V_{K}$-center. Approximately similar radiations were recorded in the TSL spectra in the temperature range of $180 \mathrm{~K}$, when the TSL peak belonging to the family of $V_{K A}(N a)$-centers is destroyed. The 
similarity of the TSL spectra in the delocalization of $V_{K}$ - and $V_{K A}(\mathrm{Na})$-centers is explained by the fact that in both cases, unrelaxed holes are mobile, which recombine with $F$-centers, while the effect of sodium located in the cation nodes of the lattice on the recombination processes is not observed.

As mentioned above, in the absence of deformation in RbI crystals under the action of X-ray radiation, $I_{3}^{-}$-centers are effectively created by the mechanism of association of mobile halogen atoms ( $H$-centers) with each other.

In $\mathrm{RbI}$ crystal, the maximum thermal destruction of the $\mathrm{I}_{3}^{-}$-centers occurs in the temperature range $360 \div 365 \mathrm{~K}$, as shown by the TSL curves (curve 1 of Figure 2). The products of thermal dissociation of $I_{3}^{-}$-centers are mobile in the specified temperature range, and they actively recombine with fixed $F$-centers.

On the example of $\mathrm{KBr}$ and $\mathrm{KCl}$ crystals, the dissociation products of $\mathrm{X}_{3}^{-}-$ centers under optical stimulation by photons with energy corresponding to the maxima of the absorption bands of $X_{3}^{-}$-centers were experimentally established $[2,25]$.

The algorithm of an interesting experimental method is as follows: the crystal is irradiated with X-rays, usually for $1 \div 2$ hours, at temperatures of $180 \div 200 \mathrm{~K}$ in order to create a maximum concentration of $X_{3}^{-}$-centers (simultaneously $F$-centers), since in the specified temperature range they are complementary radiation defects. After which the crystal is heated to $305 \div 310 \mathrm{~K}$ (close to the peak maximum of $360 \mathrm{~K}$ TSL) for annealing of low-temperature peaks related to $X_{2}^{-}$molecules $\left(H_{A}, V_{K}, V_{K A}, V_{F}\right.$-centers). Then the crystal is cooled to $80 \mathrm{~K}$ so that the dissociation products of the $X_{3}^{-}$-centers are thermally frozen, after which, at $80 \mathrm{~K}$, a powerful optical stimulation is performed for 30 minutes with photons with an energy corresponding to the maximum absorption of the $X_{3}^{-}$-centers, which leads to photodissociation of the $X_{3}^{-}$-centers.

After processing the crystal according to the above technology, TSL is recorded in order to detect the products of photodissociation of $X_{3}^{-}$- centers. According to the data $[2,25]$, the products of photodissociation of $X_{3}^{-}$-centers in AHC are $V_{K}, V_{F}$ and $H$-centers. All these hole centers, when recombining alternately with $F$-centers, may form exciton-like luminescence according to the following schemes:

$$
V_{K} \ldots F \rightarrow e^{+}+v_{a}^{+} e^{-} \rightarrow e_{s}^{0}\left(v_{a}^{+}\right) \rightarrow \alpha-\text { luminescence }
$$

or

$$
V_{F} \ldots F \rightarrow v_{c}^{-} e^{+}+v_{a}^{+} e^{-} \rightarrow e_{s}^{0}\left(v_{a}^{+} v_{c}^{-}\right) \rightarrow d-\text { luminescence. }
$$

In $\mathrm{RbI}$ crystal, the $\alpha$-luminescence has a maximum emission at $2.22 \mathrm{eV}$.

During recombination of $H \ldots F$-centers, the crystal lattice is restored. This process can proceed non-radiatively.

In the TSL spectra, a radiation band with a maximum at $2.5 \mathrm{eV}$ was recorded at a peak at $360 \mathrm{~K}$ (box $d$ of Figure 2). According to the data [12], radiation with a maximum at $2.52 \mathrm{eV}$ refers to an exciton-like formation in the divacancy field.

Based on the experimental results, we assume that during the thermal dissociation of the TSL peak at $360 \mathrm{~K}, V_{F}$-centers are released and, when recombining with $F$-centers, exciton-like luminescence forms in the divacancy field, according to reaction 9. 
Thus, according to the registration of TSL, the maxima of the fracture temperature of specific radiation defects are determined, and their recombination nature is characterized by studies of the spectral composition of TSL irradiated crystals.

In this direction, it is especially important to determine the activation energy of TSL peaks, in addition to the spectral composition and the temperature of their maximum destruction, which is the integrity of the study of thermal activation processes taking into account low-temperature elastic deformation.

TSL curves can be characterized by the temperature $T_{m}$, at which the luminescence intensity is maximum, and the half-width of the peak $\delta=T_{2}-T_{m}$, where $T_{2}$ - is the temperature on the descending branch of the thermally stimulated luminescence curve, at which the intensity reaches the value $J_{2}=0.5 J_{m}$.

The thermally stimulated luminescence curve for one type of capture centers can be approximately calculated theoretically. For the case when the probability of recombination significantly exceeds the probability of repeated localizations, the calculated TSL curve takes the following form at the capture centers:

$$
J=n_{0} p_{0} e^{-\frac{E_{t}}{k T}}\left(-\frac{p_{0}}{\beta} \int_{T_{1}}^{T_{2}} e^{-\frac{E_{t}}{k T}} d t\right)
$$

where $n_{0}$ - the concentration of ionized centers of luminescence at the time of the beginning of heating of the crystal, equal to the concentration of localized charges; $p_{0}$ - frequency factor; $E_{t}$ - activation energy of the depth of the capture centers; $\beta$-sample heating rate; $k=8.63 \cdot 10^{-5} \mathrm{eV} / \mathrm{K}$ - Boltzmann constant.

Determination of the depth of the $E_{t}$ capture centers is possible by different methods: Rendall-Wilkinson, Parfianovich, Lushchik, Urbach, AntonovRomanovsky [2, 26-29]. Some methods use individual elements of the TSL curve, others use the entire curve, and therefore have different degrees of accuracy. However, it is by the Lushchik method that it is possible to estimate the activation energy for one experiment, since in others values with different heating rates are required.

The activation energy of the depth of the $E_{t}$ capture centers was estimated using the Lushchik method. In our case, the probability of $W_{p}$ recombination significantly exceeds the probability of $W_{c}$ capture, therefore, the relation is fulfilled (see Table 1):

$$
E_{t}=\frac{k T_{m}^{2}}{\delta}
$$

Table 1.

The maximum of the breaking temperature and activation energy of some radiation defects in $\mathrm{RbI}$ crystals.

\begin{tabular}{l|l|l}
\hline Radiation defect & $\begin{array}{l}T_{m} \text { - maximum breaking } \\
\text { temperature, } \mathrm{K}\end{array}$ & $\begin{array}{l}E_{t}-\text { activation energy of the } \\
\text { depth of the capture centers, eV }\end{array}$ \\
\hline$F^{\prime}$ & 113 & 0.210 \\
\hline$H_{a}$ & 128 & 0.321 \\
\hline$V_{K}$ & 148 & 0.413 \\
\hline$V_{K A}$ & 178 & 0.531 \\
\hline$I_{3}^{-}$ & 360 & 0.822 \\
\hline
\end{tabular}


Analyzing the data in the table, it can be concluded that with an increase in the temperature of destruction of the capture centers, the activation energy of the depth of the centers increases.

\section{Conclusion}

In conclusion, we note that on the example of RbI crystal, the reinforcing effect of elastic deformation on luminescence (with a maximum at $3.9 \mathrm{eV}(\sigma)$ and $\left.3.1 \mathrm{eV}\left(E_{x}\right)\right)$ is demonstrated, the nature of which is determined by the exciton relaxation mechanism, characterized by the free path length before self-trapping.

For the first time, a deformation-stimulated recombination assembly of exciton o of such a formation is registered, manifested by tunneling $\left(F^{\prime}, V_{K}\right)$ and thermostimulation of electronic $\left(F^{\prime}\right)$ and hole $\left(H_{A}, V_{K}, V_{K A}\right.$-centers) radiation defects, effectively created during elastic deformation, is carried out at $85 \mathrm{~K}$.

Thus, an experimental method of influencing the behavior of electronic excitations is proposed to identify the nature of luminescence, based on the exciton mechanism of relaxation of electronic excitations in alkali halide crystals.

\section{Acknowledgments}

This research has been funded by the Science Committee of the Ministry of Education and Science of the Republic of Kazakhstan (Grant No. AP09057911).

\section{References}

[1] S. Song and R.T. Williams, Self-Trapped Excitons: 2nd edition (Springer, Berlin, 2013) 410 p. [Google Books]

[2] C.B. Lushchik, A.C. Lushchik, Decay of Electronic Excitations with Defect Formation in Solids (Nauka, Moscow, 1989) 262 p. [Google Books] (In Russian)

[3] Ch. Lushchik et al., Physics of the Solid State 60 (2018) 1487-1505. [CrossRef]

[4] A. Elango et al., Radiation Measurements 33 (2001) 823-827. [CrossRef]

[5] K. Shunkeyev et al., Eurasian j. phys. funct. mater. 2(3) (2018) 267-273. [CrossRef]

[6] L. Myasnikova et al., Nucl. Instrum. Meth. B 464 (2020) 95-99. [CrossRef]

[7] V. Babin et al., J. Phys. Condensed Matter 11 (1999) 2303-2317. [CrossRef]

[8] H. Nishimura et al., J. Lumin. 58 (1994) 347-349. [CrossRef]

[9] H. Nishimura et al., J. Lumin. 62 (1994) 41-47. [CrossRef]

[10] M. Kobayashi et al., J. Lumin. 48-49 (1991) 98-102. [CrossRef]

[11] K. Shunkeyev et al., J. Phys. Conf. Ser. 830 (2017) 012139. [CrossRef]

[12] V. Babin et al., J. Lumin. 81 (1999) 71-77. [CrossRef]

[13] E. Vasilchenko et al., Phys. Status Solidi (b) 174 (1992) 155-163. [CrossRef]

[14] V. Babin et al., J. Lumin. 76-77 (1998) 502-506. [CrossRef]

[15] K. Shunkeyev et al., Low Temp. Phys. 42(7) (2016) 580-583. [CrossRef]

[16] K. Shunkeyev et al., Low Temp. Phys. 45(10) (2019) 1127-1130. [CrossRef] 
[17] I. Kaplunov et al., Opt. Spectrosc. 128(10) (2020) 1583-1587. [CrossRef]

[18] I. Kaplunov et al., J. Phys. Conf. Ser. 1697(1) (2020) 012253. [CrossRef]

[19] K. Shunkeyev et al., J. Phys. Conf. Ser. 400 (2012) 052032. [CrossRef]

[20] A. Lushchik et al., Surf. Rev. Lett. 9 (2002) 299-303. [CrossRef]

[21] T. Yoshinari et al., J. Phys. Soc. Jpn. 39 (1975) 1498-1505. [CrossRef]

[22] K. Tanimuraetal., J. Phys. Soc. Jpn. 61 (1992) 1366-1379. [CrossRef]

[23] E.A. Vasil'chenko et al., Fizika Tverdogo Tela 28 (1986) 1991-1997. (In Russian)

[24] M. Ikezawa et al., J. Phys. Soc. Japan 27 (1969) 1551-1563. [CrossRef]

[25] A. Elango et al., Phys. Status Solidi (b) 78(2) (1976) 529-536. [CrossRef]

[26] R. Chen, J. Applied Physics 40(2) (1969) 570-585. [CrossRef]

[27] M. Balarin, Physics Letters 64A(5) (1978) 435-438. [CrossRef]

[28] A. Delunas et al., J. Lumin. 29 (1984) 187-197. [CrossRef]

[29] R.K. Gartia et al., J. Phys. D: Appl. Phys. 26 (1993) 858-861. [CrossRef] 